\title{
"das sie zigel machten vnd mit fewer kochten" Babylonische Verhandlungen in den Chroniken Rudolfs von Ems und Hartmann Schedels
}

\section{Babylonian presentations in world chronicles by Rudolf von Ems and Hartmann Schedel}

Johannes Deibl

\begin{abstract}
This article tries to give an insight into how medieval and early modern chronicles such as Rudolf's Weltchronik and Hartmann Schedel's Liber chronicarum capture the polysemantic topic of Babylon for their recipients. It shows that the focus lies on the description of the historic metropolis, the narration of the tower of Babel and the Babylonian exile. In some ways their modes of information transfer differ from one another. On the one hand, Schedel has a lot more to offer in terms of paratextual preparation, which put Babylon to the forefront effectively. On the other hand Rudolf separates narratives from the time of Babel onward, which means a crucial point for the structure of the text. As Schedel sets the Babylonian exile as a turning point on the macrostructure of history, Rudolf prefers to honor King David's historical impact instead. While the plot of both works is much the same, the presentations of Babylon show some shifts in comparison for the medieval recipients.
\end{abstract}

\section{Keywords}

Rudolf von Ems; Hartmann Schedel; World chronicle; Babylon; Tower of Babel; Babylonian exile; Secular history; Salvation history; Periodisation; Information transfer; 13. Century; 15. Century 


\section{Hinführung}

Kaum ein Begriff findet in geistesgeschichtlichen wie popkulturellen Belangen so ungebrochen Niederschlag wie der von Babylon. Als mesopotamische Machtmetropole der vorchristlichen Welt, namensgebendes Zentrum orientalischer Hochkultur, biblisches Sinnbild menschlicher Hybris im Turmbau zu Babel, abwertende Allegorie („Hure Babylon") oder überhaupt als Metapher für profane Lasterhaftigkeit; Babylon war und ist gleichsam Faszinosum und Reflexionsanstoß in gelehrten wie in breiten Bevölkerungsschichten.

Dieser Beitrag nähert sich Babylon anhand seiner mittelalterlichen, volkssprachlichen Aufnahme und Vermittlung an. Ein besonders breites Publikum konnte über die sogenannte Weltchronistik erreicht werden, Geschichtswerke mit umfassendem zeitlichem Anspruch und heilsgeschichtlicher Prägung. Diese Textform wurde ab dem 13. Jahrhundert „zum wichtigsten Medium der Tradierung klerikaler Wissensbestände an Laien“1 überhaupt. Was aber konnten die Rezipierenden vom Phänomen/Mythos/Sinnbild Babylon aus einer solchen Schrift entnehmen und wie sind die einschlägigen Passagen im Rahmen der Werkkonzeption zu bewerten?

Anhand zweier Werke soll eine knappe Annäherung dazu erfolgen. Herangezogen werden die Weltchronik des Rudolf von Ems in Form der Wernigeroder Handschrift ${ }^{2}$ sowie Hartmann Schedels deutschsprachige Version seines Liber chronicarum ${ }^{3}$. Die Aufbereitung erfolgt nahe an den Texten und Bildern; so soll eine Vorstellung davon vermittelt werden, wie sich der vielschichtige Topos Babylon den Rezipierenden der ausgewählten Werke mitgeteilt hat. Naturgemäß hängt diese Frage stark an der grundsätzlichen „Anlage“ der Chroniken, die bei aller Ähnlichkeit nie gleich ausfällt.

\section{Rudolfs Weltchronik}

Der herangezogene Textzeuge wird behelfsmäßig nach seinem früheren Aufbewahrungsort als Wernigeroder Handschrift betitelt. Rudolfs Projekt zur Erzählung der Weltgeschichte ist Fragment geblieben; der Text reißt bereits mit der Schilderung vom Tod Salomons ab. Eine Fortsetzung folgt in der Wernigeroder Handschrift direkt an den Text, reicht aber ihrerseits nicht über die Zeit des Propheten Elischa hinaus.

1 Herweg, Mathias (2016): Erzählen unter Wahrheitsgarantie - Deutsche Weltchroniken des 13. Jahrhunderts. In: Wolf, Gerhard [et al.]: Handbuch Chroniken des Mittelalters. Berlin - Boston: De Gruyter, S. 145180 (hier S. 145).

2 Rudolf <von Ems>: Weltchronik. Und erste Fortsetzung (sog. Wernigeroder Handschrift), BSB München, Cgm 8345, online: http://daten.digitale-sammlungen.de/ db/0008/bsb00080685/images/ (5. 10. 2019). Die den Zitaten im Text folgenden Verszuordnungen entsprechen der Ausgabe: Ehrismann, Gustav (hrsg.) (1915): Rudolf 〈von Ems〉: Weltchronik. Aus der Wernigeroder Handschrift. Berlin: Weidmannsche Buchhandlung (=Deutsche Texte des Mittelalters, Bd. 20).

3 Für diese Ausführungen wird folgender Nachdruck der Chronik zitiert: Füssel, Stephan (hrsg.) (2005): Schedel, Hartmann: Weltchronik. Kolorierte Gesamtausgabe von 1493. Augsburg: Weltbild. 
Die Weltchronik gilt mit ihrer Datierung auf die Mitte des 13. Jahrhunderts als die erste mit umfassendem zeitlichem Anspruch in deutscher Sprache. ${ }^{4}$ Es liegt ein reüssierendes Werk vor, das eine reiche Rezeptionsgeschichte - alleine über 100 handschriftliche Textzeugen sind erhalten - aufweist. Verfasst wurde es zu Ehren des Stauferkönigs Konrad IV., was auch in der Textrealisierung Niederschlag fand.

Rudolf kündigt eine „traditionelle“ Einteilung in Weltalter an - gemäß der prägenden Periodisierung des Kirchenvaters Augustinus sind es sechs Einheiten -, von der Weltschöpfung bis in die Gegenwart des Verfassers reichend. Nur ein Teil davon konnte realisiert werden. Die chronikale Erzählung übernimmt Augustinus' Denkschema der beiden civitates (civitas terrena/civitas dei), um die Heils- und Profangeschichte in eigener Weise zu komponieren (auf der Zeitleiste wiederum durch die Weltalter unterteilt).

Die Weltchronik tritt stark polyhistorisch auf, breitet eine Vielzahl an Wissensgebieten vor den Rezipierenden aus, wobei Rudolf sich an zahlreichen Quellen bedient. Ein Fokus liegt dabei auf der Historia scholastica des Petrus Comestor, der Imago Mundi des Honorius Augustodunensis sowie an historiographischen Werken Ottos von Freising.

Für lange Zeit wurde die Weltchronik in den Textwissenschaften kaum oder aber geringschätzig beäugt; es wurden die fehlende sprachliche Brillanz angeprangert - Rudolf hatte sich ganz bewusst dem sermo humilis verschrieben - wie auch die eigenständige Leistung seiner Arbeit ob der zahlreichen Quellenübernahmen in Frage gestellt. Mit dem steigenden Forschungsinteresse an der volkssprachlichen, mittelalterlichen Chronik als Textgattung wurde aber auch Rudolfs Beitrag in den letzten Jahrzehnten häufiger herangezogen und dabei ein Stück weit rehabilitiert. ${ }^{5}$

\section{Schedels Weltchronik}

Die zweite gewählte Chronik ist volksläufig als Schedelsche Weltchronik bekannt (gemäß ihrem Verfasser, dem Nürnberger Arzt Hartmann Schedel). Dem Druckort entsprechend, scheint sie auch unter der Bezeichnung Nürnberger Chronik auf, während man sie in ihrer lateinischen Version weniger distinkt als Liber chronicarum ausweist.

Das Erscheinungsjahr des Erstdrucks ist 1493, was für beide Fassungen gilt; erstellt wurden eine deutsch- wie eine lateinsprachige Chronik, wobei die „Szenegröße“ Anton Koberger für den Druck verantwortlich zeichnete. Die Konzeption richtet sich (gleich

4 Einen einführenden Überblick zu frühen chronikalen, volkssprachlichen Schriften erhält man bei Müller, Stephan (2016): Anfänge deutschsprachiger Chronistik im 11. und 12. Jahrhundert. In: Wolf, Gerhard [et al.]: Handbuch Chroniken des Mittelalters. Berlin - Boston: De Gruyter, S. 129-144.

5 Man beachte in diesem Zusammenhang etwa folgende Veröffentlichungen: Melville, Gert (2001): Die Wege der Zeit zum Heil. Beobachtungen zu mittelalterlichen Deutungen der Menschheitsgeschichte anhand der ,Weltchronik' des Rudolf von Ems. In: Barbara, Hanna [et al.]: Zeitenwende - Wendezeiten. Dresden: Thelem (=Dresdner Hefte für Philosophie, 3), S. 159-179. - Wedell, Moritz (2013): Poetische ,willekür'. Historiographie zwischen Inspiration und rhetorischer Produktion in Rudolfs von Ems ,Weltchronik'. In: Zeitschrift für deutsche Philologie 132, Heft 1, S. 1-28. - Gebert, Bent (2016): Nebenwege in der Vormoderne. Semantische Tradition und Erzählen in der ,Weltchronik' Rudolfs von Ems. In: Mittellateinisches Jahrbuch 51, Heft 1, S. $39-72$. 
der Weltchronik des Rudolf von Ems) nach dem Sechs-Weltalter-Schema und wagt einen Ausblick auf das kommende Jüngste Gericht.

Schedels Werk gilt als einer der bedeutendsten Frühdrucke, sowohl was die damaligen Absatzzahlen anbelangt, ${ }^{6}$ als auch bezüglich der heutigen Einschätzung durch die kunst- und buchwissenschaftliche Forschung. Im Vordergrund steht eine bildliche Ausgestaltung, die sich in Superlativen bewegt. Keine geringere Malerwerkstatt als die von Michael Wolgemut - zur Entstehungszeit der Nürnberger Chronik war auch Albrecht Dürer dort beschäftigt - wurde zur Anfertigung der illustrativen Holzschnitte verpflichtet. Vor allem auf die zahlreichen Stadtansichten wird bis heute zurückgegriffen, wenn es darum geht, ausgewählte Städte in einem altehrwürdigen Kleid zu vergegenwärtigen.

Aber auch der Text selbst birgt mehr als eine bloße Aneinanderreihung bereits vorhandener Quellen; er kann im Zusammenspiel mit der gleichberechtigten Bildebene die Heils- und Profangeschichte lebendig vermitteln und erweist sich in seiner Konzeption als untersuchenswert, wie Bernd Posselts rezente Publikation unter Beweis stellt. ${ }^{7}$

Schedel bedient sich an zahlreichen chronikalen Vorlagen: Hervorzuheben sind das Supplementum chronicarum des Giacomo F. Forèsti, die Vita pontificum des Bartolomeo Platina wie auch das Fasciculus temporum des Werner Rolevinck. Für die Stadtbeschreibungen bildet Enea S. Piccolominis Europa eine wichtige Quelle. Wie zu erkennen ist, werden überwiegend Quellen aus dem italienischen Raum herangezogen.

\section{Babylonische Vermittlung}

Rudolfs Weltchronik liefert eine Beschreibung der historischen Stadt Babylon, festgeschrieben in die Herrschaftszeit der sagenumwobenen Königin Semiramis. Der an der biblischen Erzählung orientierte Turmbau zu Babel wird prominent geschildert (in der Wernigeroder Handschrift auch mit einer kolorierten Zeichnung der Bauarbeiten angereichert). Das babylonische Exil wird im ersten Prolog des Werkes als Startschuss für das fünfte Weltalter angekündigt. Auch darüber hinaus fällt der Begriff Babylon wiederholt und kann dabei narrative Funktionen im Rahmen der Werkkonzeption erfüllen.

Die Beschreibung der historischen Stadt Babylon präsentiert sich vor allem unter dem Gesichtspunkt ihrer schieren Uneinnehmbarkeit:

„der stat rincmur, du drumbe gie / und Babilonie umbe vie, / was als uns du warheit seit, / an dicke funzic klaftir breit / und zwei hundirt klaftir hoch. [...] mit hundirt portin erin / sah man si wol beslozin sin / und vestecliche virspart, mit gůtin turnin wol bewart / was si und wol ze wer bestat." (3638-3642; 3648-3652)

6 Vgl. dazu etwa die zusammengetragenen Zahlen bei Green, Jonathan (2006): Marginalien und Leserforschung - Zur Rezeption der ,Schedelschen Weltchronik‘. In: Archiv für Geschichte des Buchwesens, Bd. 60, S. 184-261 (hier 188f.).

7 Posselt, Bernd (2015): Konzeption und Kompilation der Schedelschen Weltchronik. Wiesbaden: Harrassowitz (=Monumenta Germaniae Historica/Schriften, Bd. 71). 
Babylon ist nicht vorrangig Stadt, sondern Festung: Ihre Mauern wurden zur Zeit des Bestehens zu den sieben Weltwundern gezählt. Genau diesem Anspruch folgt die Textstelle, wenn sie die Exorbitanz der wehrhaften Anlage katalogartig wiedergibt. Da die Stadtbeschreibung direkt in den weniger rühmlichen Lebenswandel von Königin Semiramis übergeht, bleibt an der Metropole ein moralisch gesehen fahler Beigeschmack hängen.

Eine Konstante in der biblischen Überlieferung bildet das babylonische Exil, die Exilierung jüdischer Bürger nach der EroberungJudas/Jerusalems mit Beginn des sechsten Jhdts. v.Chr. Gemäß der augustinischen Periodisierung bildet dieser Abschnitt der Geschichte den Beginn eines neuen Zeitsegmentes. Der erste Prolog zur Chronik nimmt diese traditionsträchtige Zäsur auf: „das si gevie der Babilon / durh ir gediendin sundin lon, / damit der vierdin welte zil / ein ende nam mit wundirn vil“ (137-140).

Letztendlich wird dem babylonischen Exil diese angekündigte Sonderstellung keineswegs eingeräumt: Nach der Geburt des Propheten Samuel (gewissermaßen dem Auftakt zum neuen Weltalter) kommt die bestimmende Zeit König Davids. Für die Wahl dieser Zäsur wurden durch die Forschung unterschiedliche Beweggründe ins Treffen geführt: Mathias Herweg betont die höfisch-poetologische Verwurzelung Rudolfs, die sich unter anderem durch die makrostrukturelle Gliederung anhand der Protagonisten ausdrücke - in diesem Fall David. ${ }^{8}$ Ingrid von Tippelskirch verweist hingegen stärker auf die Neuorientierung der Textgliederung an den gegebenen biblischen Büchern. ${ }^{9}$ Beide Beobachtungen sind plausibel; die Betonung König Davids (und damit die Streichung der vorrangigen Rolle des babylonischen Exils auf der Makroebene) steht und fällt jedoch mit der Zielsetzung des Werkes, Konrad IV. in ein schillerndes Licht zu rücken. Die geschaffene Kontinuität zwischen dem biblischen David und Konrad (dem König von Jerusalem) ist Erklärung genug, den israelitischen König in den Fokus des Erzählabschnittes zu setzen.

Weshalb die augustinische Version im Prolog dennoch angekündigt wurde, wird damit kaum klarer. Plausibel scheint, dass Rudolf diese Divergenz aufgezogen hat, um die vollzogene Modifikation für den Rezeptionsakt zu markieren; im Überraschungseffekt tritt die vorgenommene Abweichung stärker ins Bewusstsein. Die doch umfangreiche Reflexion im fünften Prolog, worin der Beginn eines Weltalters nur von etwas umwälzend Neuem bedingt werden könne, legt jedenfalls nahe, dass Rudolf in dieser Frage nichts dem Zufall überlassen hat.

Neben der Beschreibung der historischen Stadt und der Ankündigung des babylonischen Exils (zu einer Realisierung kommt es bei Rudolf nicht mehr), finden sich immer wieder Einsprengsel, die Babylon aufgreifen, wie etwa folgende knappe Textstelle: „da sih liez uf die erde nidir / von Noe dú groze diet, / do si von Babilonie schiet." (30633065) Die Nachkommen Noahs zerstreuen sich auf dem Erdkreis, wobei die babylonische Verteilung der Konsequenz des frevelhaften Turmbaus zuzuordnen ist; den 72 Völkern des Turmbauvorhabens wird durch Gott die gemeinsame Sprache genommen, was das

$8 \quad$ Vgl. Herweg [Anm. 1], S. 157.

9 Tippelskirch, Ingrid von (1972): Die ,Weltchronik‘ des Rudolf von Ems. Studien zur Geschichtsauffassung und politischen Intention. Diss., Univ. Karlsruhe, S. 100. 
Projekt zum Scheitern bringt. Die hilflos gewordenen Menschen werden in alle Ecken der bekannten Welt gesetzt. Die Anspielung auf die Turmbau-Episode - der Bau selbst wird an anderer Stelle beschrieben - folgt direkt auf profane, topographische Informationen, die einander enumerativ ablösen. Während der Erdenraum für die Rezipierenden aufgeschlüsselt wird, werden die Stätten auch demographisch besetzt; die heilsgeschichtliche Episode des Turmbaus übernimmt diese Rolle schon in dieser kleinen Andeutung. Babylon (Babel) dient hier dazu, die Vermittlung weltlicher Inhalte letztlich doch mit dem biblischen Sinnangebot zu verklammern. Wie es im Verfasserlexikon heißt, „sichern leitmotivartige Querverweise die Erkenntnis von planvoller Zielgerichtetheit und Kontinuität der Entfaltung in der Welthistorie. " ${ }^{10}$ Das Babel-Einsprengsel fungiert als ein solcher Querverweis.

Bezüglich der Schedelschen Weltchronik fällt zunächst die starke Präsenz der Paratexte ins Auge. Schon bei einer Annäherung über das Register werden drei einschlägige Angaben zu Babylon vorgefunden:

- $\quad$ Babilon der thurn von weme vnd warumb er gepawt warde

- Babilonia ein hawbtstatt in Kaldea

- $\quad$ Babilon ist hie zerstort worden (o.S.)

Die Metropole wird zweimal ausgewiesen, einmal in ihrer Bedeutung und Blüte, das andere Mal in ihrer Zerstörung. Ein weiterer Eintrag verweist auf den biblischen Turmbau zu Babel und seine Umstände. Ein zielsicherer Zugriff „von außen“ auf die Vermittlung rund um Babylon scheint gewährleistet zu sein.

Die Erschließung der historischen Stadt wird - im Gegensatz zu Rudolfs Weltchronik durch die Illustrationsebene mitbestimmt. Die Darstellungen Babylons weisen im Unterschied zu vielen zeitaktuellen Stadtdarstellungen Europas im Werk keinen authentischen Anspruch auf. ${ }^{11}$ Die Textpassage selbst schlägt in dieselbe Kerbe wie jene bei Rudolf; auch hier wird der Festungscharakter der Stadt zum entscheidenden Kriterium.

Für einen gesonderten Status Babylons auf der Illustrationsebene spricht nicht nur die einmalige Verwendung der Bilder (viele der Abbildungen wurden wahllos mehrfach verwendet), sondern überhaupt die zweimalige Darstellung der historischen Stadt. Daran hängt ein weiterer Aspekt, der sich in Bezug auf Babylon erschließen lässt; ebenfalls öfter als einmal dargestellt ist Jerusalem, Babylons ewiger Antagonist. Das Gegensatzpaar des sündhaft-weltlichen Babylons und des (mitunter) himmlischen Jerusalems tritt aber nicht nur in der numerischen Sonderstellung beider Städte auf der Bildebene auf, sondern ist auch dem Rezeptionsakt eingeschrieben: Der Salomonische Tempel Jerusalems weicht durch das simple Wenden des Blattes (XVIIr/v) dem Turmbau zu

10 Walliczek, Wolfgang (1992): Rudolf von Ems. In: Ruh, Kurt [et al.]: Die deutsche Literatur des Mittelalters. Verfasserlexikon. Berlin - New York: De Gruyter, Sp. 322-345.

11 Die Aufzählung der authentischen Ansichten ist u.a. einzusehen bei Füssel, Stephan: Einleitung. In: Schedel [Anm. 3], S. 30. 
Babel - eine solche räumliche Gegenüberstellung blieb den Rezipierenden bestimmt nicht verborgen.

Abseits der durch das Register ausgewiesenen Passagen liefert die Chronik noch einen weiteren paratextuellen Babylon-Bezug. Das Werk arbeitet mit überwiegend vertikal angelegten, genealogischen Linien, die sich mal als kolorierte Leiste, mal als Rankenwerk über die Druckseiten bewegen; ein Machthaber folgt dem nächsten in Form einer typisierten Abbildung sowie dem Namen des Herrschers. In diesem Rahmen kommen auch die neubabylonischen Machthaber („Nabuchodonosor“, „Euilmerdoch“ etc.) zum Zug, deren Rollen in der Weltgeschichte im Mengentext expliziert werden. Wie bei überblicksschaffenden Marginalien wird ein rascher Informationskanal jenseits des Mengentextes angeboten und so eine zielgerichtete Aufnahme der genealogischen Gegebenheiten gewährleistet. ${ }^{12} \mathrm{Zu}$ Neubabylon ist anzumerken, dass entgegen der eigenwilligen Periodisierung bei Rudolf hier das gewohnte System nach Augustinus vorherrscht: Das babylonische Exil tritt als eröffnende Zeitspanne eines neuen Segmentes auf und wird im Gegensatz zu Rudolfs Chronik auch noch ausgeführt.

Für die prominenteste Episode, die Erzählung des Turmbaus zu Babel (Gen 11,1-9), wird eine direkte Gegenüberstellung der beiden Chroniken vorgenommen: Beide Textversionen werden von einem illustrierten Turm begleitet (bei Rudolf nicht in jedem erhaltenen Textzeugen der Fall $)^{13}$, doch nur bei Schedel erhält die Passage einen Zwischentitel, der ihre Wichtigkeit im Rahmen der Heilsgeschichte markiert. Bis dahin findet sich eine solche Kennzeichnung nur vor der Arche Noah-Textstelle wieder, erst wesentlich später im Werk tritt sie inflationär auf.

Zu den Textstellen: Augenscheinlich wird im direkten Vergleich, dass der Turmbau unterschiedlich motiviert dargestellt wird - konkret anhand der Finalsätze.

„do geriet in Nemrot, / ob si das gerne woltin / fúrkomin, das si soltin / mit so rilichin sachin / einen turn in machin / der inden himil gienge / des ho̊he in das virvienge / das si decheines wazers flůt / irreichen mohte." (Rudolf, 1227-1235)

„vnd das er die menschen von gottes forcht abforderte so riet er [Nimrod] in das sie zigel machtē vn̄ mit fewer kochten vnd einen hohen thurn paweten des gipfel od hoehe bis an den himel ruerte. gleich als wuerden sie dar durch steigen in den himel." (Schedel, XVIIIr) ${ }^{14}$

12 Verglichen wird eine solche Marginalien-Gliederung mitunter mit „eine[r] Art Schnellspur“, siehe Baumert, Andreas [et al.] (2016): Texten für die Technik. Leitfaden für Praxis und Studium (2. Aufl.). Berlin Heidelberg: Springer, S. 191.

13 Vgl. Kratzert, Christine (1974): Die illustrierten Handschriften der Weltchronik des Rudolf von Ems. Diss., Univ. Berlin, S. 68.

14 Dieser Textstelle wurde der Beitragstitel entnommen („das si zigel machtē vn mit fewer kochten“); wie die Nachkommen Noahs Stein um Stein aufbereiteten, um etwas Totales zu schaffen, so erinnert der Umgang der mittelalterlichen Chronisten mit vorgefundenen Versatzstücken alter Quellen an diesen Arbeitsweg. Auch hier kann letztlich das Resultat niemals dem allumfassenden Vorhaben gerecht werden, wenngleich einige Versuche dazu immerhin schriftlich vorliegen. 
Bei Rudolf wird der Turmbau explizit als Präventivmaßnahme gegen eine etwaige weitere Sintflut ausgewiesen. Schedel verzichtet auf diesen „materialisierten“ Grund und räumt stattdessen unvermittelt mit der Gottesfurcht auf; die Sintflutepisode liegt an diesem Punkt der Ereignisabfolge allerdings nahe genug, um auch bei Schedel implizit mitzuschwingen. Wichtig scheint in diesem Zusammenhang, dass der Sintflut-Akzent in der Babel-Narration schon bei alten weltlichen Autoritäten auftaucht (etwa in Flavius Josephus' Jüdischen Altertümern) $)^{15}$, während die Bibelpassage diesen ausspart. Beda Venerabilis wird bei Schedel ausgewiesen und die Erzählung damit sichtbar in eine Autoritäten-Tradition gestellt; in welchem Duktus die Information in den Chroniken an die Rezipierenden gebracht wird, hängt stark davon ab, woraus der Verfasser sie schöpft. Dieser trivial wirkende Umstand zeichnet die Gattung der mittelalterlichen Weltchronik in besonderer Weise aus und bleibt auch für die Vermittlung von Babylon/Babel nicht ohne Konsequenz.

Bei Rudolf konstituiert die Textstelle zu Babel aber auch die Gesamtkomposition; ab der Turmbauepisode fächert sich die Erzählung in mehrere Stränge auf, die erst kurz vor dem Abbruch des Werkes wieder zusammenfinden. ${ }^{16}$ Verliert das babylonische Exil den Status einer Zäsur der Weltgeschichte, so wirkt die Turmbaupassage als ein willkommener Startschuss zur Abänderung des Erzählschemas.

\section{„Babylon" bei Rudolf und Schedel}

Die Weltchroniken von Rudolf von Ems und Hartmann Schedel stellen ein ganzes Bündel an Informationen rund um den vieldeutigen Begriff Babylon bereit, wovon hier exemplarisch berichtet wurde. Auch wenn beide Werke die grundsätzlich selbe Geschichte (die Weltgeschichte) erzählen, lassen sich gewisse Aspekte der Babylon-Thematik verschoben wahrnehmen, wie die folgende Rekapitulation der beschriebenen Aspekte verdeutlichen soll:

Babylon taucht in beiden Werken als historische Stadt auf. Der Fokus wird dabei jeweils auf ihre überwältigend-wehrhafte Befestigung gelegt. Bei Rudolf erhält die Stadt keine illustrative Unterstützung, während Schedel sie typisiert in ihrer Blüte und ihrem Verfall darstellen lässt. Die bildliche Ebene bei Schedel verdeutlicht dabei nicht nur den hohen Wert der Stadt im Lauf der Weltgeschichte (zweifache Illustrierung/einfache Verwendung der beiden Realisierungen im Werk), sondern betont damit ebenso den Antagonismus zwischen Babylon und Jerusalem mittels simpler Techniken (mehrfache Abbildung auch von Jerusalem; Turm von Babel folgt Salomonischem Tempel durch Umblättern).

15 Heinrich Clementz (hrsg.) (1990): Flavius Josephus: Jüdische Altertümer. I. Band, Buch I bis X (10. Aufl.). Wiesbaden: Fourier, S. 31f. Die quellentechnische Heterogenität einer chronikalen Sammlung wie der Weltchronik des Rudolf von Ems wurde von Manfred Kern in ihrem Anspruch einer vollständigen schriftlichen Darlegung der Welt betont (Kern, Manfred (2006): Welt aus Fugen. Textuelle Heterogenität in der mittelalterlichen Weltchronistik am Beispiel Rudolfs von Ems. In: Spracherwerb: generativ - interaktiv (=Zeitschrift für Literaturwissenschaft und Linguistik 36, 3), S. 123-136.).

16 Vgl. hierzu auch Herweg [Anm. 1], S. 150. 
Der Turmbau von Babel (orientiert an der biblischen Narration) ist in beiden Chroniken prominent vertreten. Im Vergleich zum biblischen Text nehmen diese Versionen mehr an Textraum für sich in Anspruch. Im direkten Abgleich der beiden Passagen lassen sich feine Unterschiede erkennen; knapp wurde gezeigt, dass bei Rudolf die Furcht vor einer neuerlichen Sintflut als Motivationsschub auftaucht (wie auch schon bei Flavius Josephus), während Schedel die Abkehr von Gott expliziert, ohne dabei die Wasserkatastrophe aufs Tapet zu bringen. Auch die Vermittlung Babels in den Chroniken hängt stark von den Quellen ab, aus denen geschöpft wird. Bei Rudolf erhält die Textstelle darüber hinaus konstitutive Bedeutung für das weitere Erzählschema; ab hier etablieren sich einzelne Handlungsstränge, die erst kurz vor dem Abbruch des Textes wieder zusammenfinden. Die Babel-Wirren zeigen sich „kontrolliert“ also auch daran, wie der Text die Folgeinformationen für die Rezipierenden aufbereitet.

Das babylonische Exil wird bei Schedel als prägender Zeitabschnitt jüdischer Geschichte eingeordnet und fungiert in bester augustinischer Tradition als Zäsur auf der Makroebene. Während Rudolf diese Bestimmung zunächst ebenfalls ankündigt, nimmt er den Einschnitt letztlich zugunsten einer Betonung der Figur König Davids vor. Gerade die Periodisierung der Welt- und Heilsgeschichte bleibt analog zur Gliederung der jeweiligen Chronik potenziell im Gedächtnis der Rezipierenden bestehen. Ob das babylonische Exil als ein historischer Einschnitt oder als eine Episode unter vielen wahrgenommen wird, bildet einen wesentlichen Unterschied, der sich aus einer differenten Vermittlung ergibt. Die Entscheidung gegen das babylonische Exil als Zäsur im Falle Rudolfs (aufgrund der gezogenen genealogischen Linie zwischen David und Konrad IV.) verdeutlicht auch den profanen Antrieb, der hinter der Umgewichtung einer so wirkmächtigen geschichtlichen Ereignisspanne stecken kann. Während das Exil bei Schedel im Rahmen des abgeschlossenen Werkes noch realisiert wird, kommt es in der Fragment gebliebenen Chronik Rudolfs dazu nicht mehr.

Die Herrschergenealogien der Geschichte drängen bei Schedel durch die illustrierten Stränge und Balken unmittelbar in den Vordergrund der Buchseiten. Auf diese Weise können auch die neubabylonischen Könige als solche rasch wahrgenommen und einfach memoriert werden. Gerade dieser Zusatz zeugt davon, welchen großen Stellenwert die Techniken der Vermittlung gerade bei Schedel spielen.

\section{Quellen und Literatur}

\section{Quellen/Textausgaben}

Clementz, Heinrich (hrsg.) (1990): Flavius, Josephus: Jüdische Altertümer. I. Band, Buch I bis X (10. Aufl.). Wiesbaden.

Ehrismann, Gustav (hrsg.) (1915): Rudolf <von Ems>: Weltchronik. Aus der Wernigeroder Handschrift. Berlin (=Deutsche Texte des Mittelalters, Bd. 20).

Rudolf <von Ems>: Weltchronik. Und erste Fortsetzung (sog. Wernigeroder Handschrift), BSB München Cgm 8345, online: http://daten.digitale-sammlungen.de/ db/0008/bsb00080685/ images/ (5. 10. 2019). 
Füssel, Stephan (hrsg.) (2005): Schedel, Hartmann: Weltchronik. Kolorierte Gesamtausgabe von 1493. Augsburg: Weltbild.

\section{Sekundärliteratur}

Baumert, Andreas [et al.] (2016): Texten für die Technik. Leitfaden für Praxis und Studium (2. Aufl.). Berlin - Heidelberg.

Füssel, Stephan (2005): Einleitung. In: Schedel, Hartmann [et al.]: Weltchronik. Kolorierte Gesamtausgabe von 1493. Augsburg, S. 1-37.

Gebert, Bent (2016): Nebenwege in der Vormoderne. Semantische Tradition und Erzählen in der ,Weltchronik‘ Rudolfs von Ems. In: Mittellateinisches Jahrbuch 51, Heft 1, S. 39-72.

Green, Jonathan (2006): Marginalien und Leserforschung - Zur Rezeption der ,Schedelschen Weltchronik'. In: Archiv für Geschichte des Buchwesens, Bd. 60, S. 184-262.

Herweg, Mathias (2016): Erzählen unter Wahrheitsgarantie - Deutsche Weltchroniken des 13. Jahrhunderts. In: Wolf Gerhard [et al.]: Handbuch Chroniken des Mittelalters. Berlin; Boston, S. $145-180$.

Kern, Manfred (2006): Welt aus Fugen. Textuelle Heterogenität in der mittelalterlichen Weltchronistik am Beispiel Rudolfs von Ems. In: Spracherwerb: generativ - interaktiv (=Zeitschrift für Literaturwissenschaft und Linguistik 36, 3), S. 123-136.

Kratzert, Christine (1974): Die illustrierten Handschriften der Weltchronik des Rudolf von Ems. Berlin.

Melville, Gert (2001): Die Wege der Zeit zum Heil. Beobachtungen zu mittelalterlichen Deutungen der Menschheitsgeschichte anhand der ,Weltchronik‘ des Rudolf von Ems. In: Barbara, Hanna [et al.]: Zeitenwende - Wendezeiten. Dresden (=Dresdner Hefte für Philosophie 3), S. 159-179.

Müller, Stephan (2016): Anfänge deutschsprachiger Chronistik im 11. und 12. Jahrhundert. In: Wolf, Gerhard [et al.]: Handbuch Chroniken des Mittelalters. Berlin - Boston, S. 129-144.

Posselt, Bernd (2015): Konzeption und Kompilation der Schedelschen Weltchronik. Wiesbaden (=Monumenta Germaniae Historica/Schriften, Bd. 71).

Tippelskirch, Ingrid von (1972): Die ,Weltchronik“ des Rudolf von Ems. Studien zur Geschichtsauffassung und politischen Intention. Karlsruhe.

Walliczek, Wolfgang (1992): Rudolf von Ems. In: Ruh, Kurt [et al.]: Die deutsche Literatur des Mittelalters. Verfasserlexikon 8. Berlin - Boston, Sp. 322-345.

Wedell, Moritz (2013): Poetische ,willekür'. Historiographie zwischen Inspiration und rhetorischer Produktion in Rudolfs von Ems ,Weltchronik‘. In: Zeitschrift für deutsche Philologie 132, Heft 1, S. 1-28.

Johannes Deibl, BA MA / johannes.deibl@stiftmelk.at, johannes.deibl@univie.ac.at

Stiftsbibliothek Melk

Abt-Berthold-Dietmayr-Straße, 3390 Melk, AT

This work can be used in accordance with the Creative Commons BY-SA 4.0 International license terms and conditions (https://creativecommons.org/licenses/by-sa/4.0/legalcode). This does not apply to works or elements (such as image or photographs) that are used in the work under a contractual license or exception or limitation to relevant rights 